\title{
Economic context analysis in mental health care. Usability of health financing and cost of illness studies for international comparisons
}

\author{
L. Salvador-Carulla ${ }^{1 *}$ and P. Hernández-Peña ${ }^{2 *}$ \\ ${ }^{1}$ PSICOST Research Association, Plaza de San Marcos 6, Jerez 11403, Spain \\ ${ }^{2}$ World Health Organisation, 20, Av Appia, CH 1211 Geneva 27, Switzerland
}

\begin{abstract}
This paper discusses an integrated approach to mental health studies on Financing of Illness (FoI) and health accounting, Cost of Illness (CoI) and Burden of Disease (BoD). In order to expand the mental health policies, the following are suggested: (a) an international consensus on the standard scope, methods to collect and to analyse mental health data, as well as to report comparative information; (b) mathematical models are also to be validated and tested in an integrated approach, (c) a better knowledge transfer between clinicians and knowledge engineers, and between researchers and policy makers to translate economic analysis into practice and health planning.
\end{abstract}

Key words: mental health care, economic analysis, international comparison, cost of illness.

\section{Background}

The disease burden estimates allocate two-fifths of life with disability to mental health needs (WHO, 2000). One in four visitors to the health systems exhibits an untreated mental health need. In low per capita income countries, resources devoted specifically to mental health interventions embrace only around 1\% of the expenditure on health; in middle per capita income countries the amount is also low (around $2.5 \%$ ). Only a few high per capita income countries spend at least $10 \%$ or more of their health dollar in mental health disease (WHO, 2008a, b; Euro Observer, 2009). Mental health care deserves special attention due to the large uncovered needs, to the lack of specialised skilled workforce and of financial resources, as well as to the complexity of the care delivery system, associated with the diversity of needs, and an interface with social care. Many problems faced in mental health economics are central to long-term care (LTC) for chronic medical conditions or for persons with disabilities (Salvador-Carulla et al. 2006). Mental health care thus constitutes a genuine paradigm of integrative medicine, as already pointed out decades ago (Eisenberg, 1973).

Opinions expressed in this paper do not reflect the position of institutions with which the authors are related.

* Address for correspondence: Luis Salvador-Carulla, PSICOST Research Association, Plaza de San Marcos 6, Jerez 11403, Spain.

(Email: luis.salvador@telefonica.net).

Patricia Hernández-Peña, World Health Organisation, 20, Av Appia, CH 1211 Geneva 27, Switzerland.

(Email: hernandezp@who.int)
Expanded service coverage requires a performing information system that sustains effective stewardship. How should programme leaders mobilise and allocate financial resources? Should policy makers direct mental health towards market services, supported by subsidies, price and product control, regulations and sanctions? Which associated determinants facilitate technical information for mental health experts, health accountants and planning officers?

Two groups of analyses that are expected to contribute to capture the effort on mental services are:

(i) different methods to analyse the costs, and impacts of alternatives, interventions or programmes, endogenous to the interventions (cost consequence and cost offset), as well as quasi-experimental or experimental programmes (cost-effectiveness, cost-utility and cost-benefit analyses);

(ii) top-down modelling reviews (Evers et al. 2007).

This note focuses on Health Financing of illness (FoI) and accounting, Cost of Illness (CoI), and Burden of Disease (BoD) and modelling studies. These analyses provide information susceptible to contribute to health planning and evidence-based management monitoring and evaluation to initiate a diagnosis of the situation and to guide priority setting, on resource allocation, systemic reform, expenditure control and systemic surveillance, major steps to intervene in the field.

\section{Towards a monitoring information system}

The mental health financing field lacks cumulated evidence to analyse and to improve the current situation. 
Recent balance between demand and the desire expressed almost everywhere to expand mental health' population coverage and actual implementations, a new momentum to ensure that health financing planning contributes to generate and to the effective, efficient, and equitable use of fresh resources (WHO, $2008 a, b$ ). Informed decisions require a monitoring system that operates an early response to adjustments needed, notably in an environment of such scarcity of resources. Health information systems (HIS), including its mental health component, should aim at a standardised baseline monitoring indicators, describing, explaining, sustaining, planning, forecasting or simulating:

- needs (including BoD studies),

- the financing, allocation and resource use in the field (FoI and Health Accounts),

- the operation and coverage of interventions through their outcomes (CoI).

The monitoring system for a huge reordering effort involves an effective change in the coverage and in the epidemiology of mental health, and requires a cohesive approach that considers the process, outputs and outcomes, which identifies the tracking indicators following a reflection on relevant operational objectives. The prerequisite of handling unsolved questions in health economics information includes the existing trend of a fragmented analysis and the adoption of standardised content and methods. A complementary effort might involve the dissemination of agreed classifications, methodological guidelines and best practice protocols.

Standardisation of the various tools includes the level of applicability, at micro- or at meso-level, as well as expected reporting practice relating to the economic and service context where these services and interventions take place. A programme and intervention are positioned within larger health organisations and territories and do not operate in a vacuum (Whitten \& Adams, 2003). Filling this 'geographical vacuum' contributes to understanding the economical effects of interventions, the generalisation of results and transfer of evidence to policy and planning. This information is critical in itself and to understand any other evidence in health economics. Territorial comparisons are particularly important in the European Union for several reasons, mainly due to patient mobility, health safety and harmonisation, as well as for the eventual convergence of member states towards an integrated health market. Cross-country comparisons have been set up as a priority for the analysis of health-care reforms and monitoring in Europe (WHO, 1996).

\section{Health financing, accounting and FoI analysis}

Health financing focuses on the strategies to collect, manage and allocate resources in the health-care system to steer and to perform interventions, while ensuring the quality of mental care, and their impact on population and patients. Expenditure data are a prerequisite to understand, describe and foresee these processes (see Table 1 above). Health-accounting studies play a relevant role, either at organisation level (Trogdon et al. 2007) or at national or international level (Poullier \& Hernandez, 2001; Thomson et al. 2009). To date, health-accounting analysis is considered to be the most comprehensive source of expenditure information for comparative purposes. The main advantages of an accounting reporting system apply also to Mental Health, as summarized in Table 1.

The 'finance-of-illness' studies display complementary information about the proportion of total health expenditure attributed to a specific illness or of a group of illnesses to facilitate comparisons across population groups and are relevant for policy planning and allocation of resources. Expenditure is disaggregated by component, thus knowing their relative importance; for example, to identify the contribution of Pharmaceuticals in total expenditure of a health condition, and to compare it internationally to adjust planning. Moreover, financing can be studied longitudinally in order to facilitate a better understanding of the evolution of (for example) pharmaceutical expenditure or the expenditure on residential care with respect to ambulatory care, issues which in mental health can indicate the development of deinstitutionalisation. It also allows us to understand the path of health expenditure within a system (e.g. public vs. private provision) or across different systems (e.g. health, social care and housing systems). Looking across systems is especially important in view of the often wideranging impacts of mental health problems, and because of differences between systems in relation to financing structure, incentives, eligibility and allocation. In summary, FoI studies contribute to handle barriers and incentives for the development of care and health policy (McDaid et al. 2006).

\section{Challenges of FoI}

Few countries prepare HA on a continuous basis. Standard methods are not implemented and the metadata lack the detail required for apprehending their content; thus, the final estimates at the country level show huge disparities; international comparisons are virtually meaningless in some classes. Current operational disparities in setting boundaries relate, for instance, to social care linked to Long-Term Care 
Table 1. Potential uses of mental health accounts

\begin{tabular}{ll}
\hline Axes under study & \\
\hline General & Monitoring and evaluation of interventions \\
& Identification of financing intervention areas \\
& Design and simulation of appropriate interventions \\
& - Resource generation \\
& Revenue collection \\
Financing & Fund pooling/mobilisation \\
& Purchasing arrangements \\
& - Resource spending \\
& Effective consumption strategies \\
& Allocative decisions \\
& Distributive policies \\
& Volume, composition and pricing of services supplied \\
& Characteristics of provider setting \\
& For example hospital based/ambulatory and community/home based \\
& Accountability of inputs and production factors (e.g. human resources on health, pharmaceuticals) \\
Provision & Inappropriateness, waste, economies of scale, efficiency gains \\
& Balanced satisfaction of beneficiaries \\
& Nature of services consumed anticipation (e.g. preventive/curative (including incentives)) \\
Consumption by disease and population groups & Equity, effectiveness, efficiency, sustainability, productivity, decentralization, \\
Indicator linkages & resource allocation, prices \\
\hline
\end{tabular}

Source: Adapted from Poullier \& Hernandez (2001).

(LTC), which affects the comparability on the share to mental health spending. Indicators on inpatient and outpatient care are in practice not standardized in their content. Moreover, the preventive-curative split is not reliable as a comparative measurement to be applied to mental health numerators. As it happens, in evidence-based medicine, quality ratings should be developed to enhance like-with-like comparisons.

The sources of information of the tools under discussion are related to their underlying classifications. In mental health, there is no single class collating all the relevant contents and there is not yet a consensus on one standard reporting format to reach full comparability of the study results. For example, some studies aggregate bipolar disorders and depression, whilst other studies aggregate anxiety disorders and depression whereas they exclude bipolar disorders from the groping. The Global Burden of Disease study (GBD) aggregates the International Classification of Diseases (ICD-10) codes in major classes or parent categories. Dementia and Alzheimer appear in different groups of diseases at ICD-10, while GBD includes both in the same group and allows for a better measurement of the mental health burden. Mental health conditions are frequently chronic and related to LTC due to their disabilities. The usual measurement strategy involving 'value $=$ price $\times$ quantity' has to be adjusted to the degree of dependency in LTC quantity. A similar case for inpatient care involves length of stay to handle a package of services consumed by day.

The extent to which disability and interventions should be covered in the costing and expenditure measurement varies also among studies, as well as the data sources and methods used in the estimates. Due to lack of appropriate records to measure disease and program interventions, specific resources at the level of detail required, cost and spending data are mixed. Cost and expenditure are sometimes understood as complementary concepts, equivalent or being one part of the other, pending on the level of analysis, e.g. expenditure is part of social cost but at micro level cost reflects the value of resources expected to be used whereas spending reflects real amounts channelled to purchase services or inputs to produce them. Some costs are not spending and vice versa. Mixture of approaches may require specific adjustments based on the content of the data at hand. Reporting complexities are also involved as providers and financing funds may change according to the type of beneficiary. The criteria to measure costs and expenditure are not standard and face feasibility problems. More explicit conceptual and methodological guidelines to measure and to report are to be promoted.

Measurement problems identified at country level are even larger when estimates refer to a single group of health conditions, such as mental disorders. 
The disparities show up in the accuracy and in the meaning of the information provided; for example, regarding the mental health share in European countries of total public health expenditure (European Commission, 2005), as well as worldwide (Saxena, 2003). Problems on comparability of expenditure have been described while testing the first proposal of a standardised manual on health expenditure (Orosz \& Morgan, 2004). A revision on what to measure and how to measure it has been initiated by Eurostat, OECD and WHO and is expected by mid 2011 (OECD, 2009), which will include classifications and guidelines, as well as recommendations on how to measure expenditure by disease.

It may be also convenient to differentiate 'frame' financing studies from accounting analyses. The purpose of frame studies is advocacy, they are frequently based on existing estimates not fully standardised, to profile a health policy. Health planning and monitoring require actual financing studies to guide evidencebased decision making.

\section{Cost-of-illness analysis}

It aims to quantify in monetary units health and nonhealth resources related to a health condition, including the impacts on non-service parts of the economy (such as lost productivity and unpaid time of family carers). This approach adopts a broader perspective than expenditure, in particular through its inclusion of the opportunity costs of non-marketed items. The information provided is linked to prevalence estimates (or, less commonly, incidence estimates), which adopt a societal perspective with insights into the distribution and the use of resources and their associated costs across all relevant sectors (e.g. employment, justice, education, special accommodation, among others). In addition, the distribution of direct and indirect costs across different illnesses or groups of illnesses can be analysed, which allows exploration from a different perspective of the total cost distribution (e.g. the proportion of the total cost attributable to psychopharmaceuticals). It is widely accepted that cost-of-illness studies from a societal perspective and prevalence-based design are indispensable for evidence-based health decision making (Saxena, 2003; Hu, 2006; Trogdon et al. 2007).

In spite of its long history dating from mid 1960s (Rice, 1996), the usefulness of CoI studies have been contested, as these studies 'may not have a clear economic meaning', as 'by focusing on health sector spending and lost labour productivity only, CoI studies provide only a very partial picture of the true macroeconomic impact of disease, and fail to consider the contribution of depleted capital accumulation, investment in human capital and demographic change to diminished economic growth'. A more general and dynamic assessment of forgone consumption opportunities was recently recommended (WHO, 2009; Chisholm et al. 2010). However, the problem of data comparability is frequently ignored and overrated the usability of $\mathrm{CoI}$ for territorial comparisons at macroand meso-level, by not taking into account the different types of analysis listed above and the existing links between macro- and microeconomics in the analysis of health care. The low quality of international CoI studies in mental health (Andlin-Sobocki et al. 2005) and its extensive use in the literature is contradictory.

\section{Modelling in mental health-care (MH) financing}

The development of models of $\mathrm{MH}$ financing and related areas may contribute to overcome some of the challenges described above. In addition to mathematical models, expert-based modelling plays a relevant role in financing, cost control and analysis of incentives and barriers at the three levels in Europe (national, regional and local).

\section{Expert-based modelling}

Mental health-care systems and networks are extraordinarily complex even at a programme level (Byng et al. 2008). The arrangements of mental health vary, not only from country to country but also across small health areas within a single region. The Mental Health Economics European Network (MHEEN-II) study provided examples of this geographical variability and on the difficulty of setting up international comparisons in very simple indicators such as the share of mental health expenditure in relation to the total public expenditure in health (Knapp et al. 2008). In order to facilitate international comparisons on financing, cost control strategies, equity, parity, effectiveness and quality of care, it is necessary to provide a definition and a model of care, which may be compared across territories. For example, it should be decided as to whether social services will be included in the model, specialised services for drug abuse, child and adolescent mental health, psychogeriatrics, or services for persons with intellectual disabilities and mental health problems. These inclusion and exclusion criteria are necessary as some countries include drug addiction services as a category for funding and provision, which is separated from $\mathrm{MH}$, while services for dementia may be included in the MH chapter of health expenditures, and there is no standard 
recording system for LTC and the various social care services related. However, half of the interventions are social and hardly should be left out. Therefore, exclusion and inclusion criteria should be agreed and a basic care model should be described to enhance meaningful international comparisons. As an example, a basic mental health community care (B-MHCC) model was defined by experts in order to rate the efficiency and appropriateness of a sample of small mental health areas in different regions (autonomous communities) in Spain (Salvador-Carulla et al. 2007). This model comprises a reduced set of 12 input and output indicators of residential and day care that provide an appraisal of the mental health care at area level. It was applied in Chile to compare service provision in Chile and Spain, also at small health area level (Salvador-Carulla et al. 2008). Expert-based models of the processes of care have been used to estimate activity based costing (ABC) of mental health care in Navarra (Spain) (Moreno et al. 2008). Financial flows should also be modelled to gauge funding and financing of the health systems.

\section{Mathematical modelling}

WHO has recently provided a frame of modelling strategies to capture the different consequences of disease guided by the question that is being asked. The methodological approaches are divided into six modelling types taking into account whether the focus is provided on microeconomics or macroeconomics, and whether non-market losses are included or not (WHO, 2009). These models include: (1) inter-temporal choice models (using output-based approach to measure losses); (2) willingness-to-pay valuation techniques; (3) simulation-based computable general equilibrium models; (4) simulation-based calibration models; (5) regression-based growth models; and (6) full-income models using estimated value of statistical life (VSL) to years lost due to disease or injury. The WHO report recommends that empirically based estimates of market losses and hypothetically based estimates of foregone welfare be separately identified and reported.

The efficiency and financing in mental health care could also be explored using regression or Bayesian multilevel analysis, knowledge discovery from data (KDD) and data envelopment analysis (DEA) (Salvador-Carulla et al. 2006). As an example, there is extensive literature about the efficiency of a set of hospitals or other health services using different model techniques, mainly ordinary least square (OLS) regression models, fixed-effect models and DEA. While OLS tend to show that hospitals with more staff have better outcomes, fixed-effect models greatly reduce potential omitted variable bias, by incorporating hospital dummies in the regression that would control for observable and unobservable differences in other factors across hospitals. On the other hand, DEA is a non-parametric method used to inform on the relative efficiency of care systems in different territories including both regional and health small areas such as primary care areas or community mental health areas (Salvador-Carulla et al. 2007). DEA modelling can be run with different inputs and outputs; it is not necessary to test a relationship function between parameters and it does not depend on the scale of analysis. Unfortunately, great disparities may be found between DEA results applied to the same set of data mainly due to different approximations to the framework defined by the expert knowledge, interferences in the information transfer between clinicians and operations analysts, the discretionary selection of input and output parameters when the number of units of analysis is small, the different modelling procedures that can be used, and the uncertainty derived from lack of data on the validity and reliability of the DEA model. These problems may be solved by combining Monte Carlo and DEA and by incorporating implicit knowledge of the experts into the model.

Apart from analysis of territories (countries, regions or local areas), modelling could be applied to estimate unit costs under conditions of high uncertainty. Fixed-effect models and Monte Carlo have been suggested as alternatives for assessing costs across territories.

It is also necessary to develop common standards and guidelines on the use of modelling for mental health-care financing and related analyses as well as to design a standard approach to incorporate implicit knowledge into the analysis of costs and financing in mental health care. Furthermore, the increasing complexity of these tools may widen the knowledge gap between clinicians and knowledge engineers on the one side, and between researchers and policy makers on the other. Finding bridges across users, clinicians, researchers, knowledge engineers and policy makers is critical to translate economic analysis into practice and health planning.

\section{BoD analysis}

BoD studies use a global morbidity-mortality indicator and adjust life years according to disability or quality of life for each illness. They measure, for example, the number of years lived with disability or impaired quality of life. They help establish a 'classification table' of diseases and facilitate comparisons within the health sector both at a national and 
international level. Health Utility Index, Health Adjusted Life Years, Quality of Life Adjusted Life Years and Disability Adjusted Life Years are different measures that can be used in BoI as well as in cost-utility analysis. The advantages and caveats of every approach have been extensively analysed in the literature (Gold et al. 2002; Ayuso-Mateos et al. 2006) and would not be treated here. The consideration of the relevance of mental health needs displayed by BoD measurements would certainly impact the amount required for expenditure on health as well as to guide allocation of resources, both national or those linked to aid, key questions in all environments (Health Affairs, 2007; Savedoff, 2007).

\section{Consensus and knowledge base for Mental Health studies in Europe}

The European Commission funded the project Mental Health Economics European Network (MHEEN) which provides a useful experience on the caveats and alternatives to improve current information problems for international comparison of mental health financing in Europe (Knapp et al. 2008). MHEEN also developed a basic standard system and terms for gathering this information and constitute a useful knowledge-base to facilitate information transfer in Europe. A relevant outcome of this project was the need to differentiate between a general and broad description of the financing systems at country level and specific studies on incentives/barriers or in the comparability of cost control mechanisms in different EU countries. A multi-level approach should be followed completing information at national level, regional level and, whenever possible at local or small health area level. These three levels should allow comparisons like-with-like. For example, it may be feasible to compare Italy, France and Spain, but Luxembourg could be also compared with smaller territorial units (i.e. country regions). Also, indicators in Luxembourg should consider the discrepancy on population denominators due to commuters, for which there are no standard adjustments recommended. Some agreement on the level of reporting should also be reached. These conditions involve setting and operating recommendations in the HIS.

On the other hand, putting together national and regional data adds confusion to the health planner as it hampers comparisons like-with-like. The WHO Report on Policies and practices for mental health in Europe is a good example of how complex territorial comparison is in current Europe, and the little use of showing in the same table information from the region of Murcia (Spain), France or Russia (WHO, 2008).
Moreover, information at small health areas may provide bottom-up analysis of the health financing and funding flows, providing relevant and complementary information gathered at the national level using a top-down approach.

MHEEN has also provided a frame analysis of the current incentives and barriers of mental health financing as well as a first assessment of cost control tools in mental health care in Europe (McDaid et al. 2006; Knapp et al. 2007). To sum up, current cost control tools such as Diagnostic Related Groups (DRGs) show usability problems in mental health care. DRG tariffs area geared to study short-term, acute episodes and they are ill suited for chronic, repetitive uses. DRGs are better suited for one off medical intervention, not repeated ones, and better suited for disease but not disabilities. Thus, they have not fully taken into account all of the costs associated with chronic mental health problems, and, in spite of further adjustments in countries like Spain or Austria, they may do little to tackle the continued under-funding of mental health services (McDaid et al. 2006). As matter of fact, DRGs have not only being criticised as being inappropriate for reimbursement, but they also may provide perverse incentives to reduce the quality of care which may not appear at the point of discharge but rather in the long run (Zechmeister \& Osterle, 2006). Other alternatives such as the use of $A B C$ in Spain (Moreno et al. 2008), the Healthcare Resource Groups (HRGs) in the UK (Mogyorosy \& Smith, 2005) or the Secondary Classification - Psychiatry (SK-PSYK) in Norway (Heurgren et al. 2004) need further revision (Northcott \& Llewellyn, 2004), particularly in mental health care as well as additional studies at the international level.

\section{The need of a complementary use of the tools}

As the MHEEN project suggests (Knapp et al. 2008), there is room for many improvements in the measurement field. In spite of the potential value of these tools, there is a widespread recognition of the problems of available data in mental health economics (Jones et al. 2007), as well as the need for more information, particularly with regard to international studies.

The different approaches to the economic context of care interventions provide complementary information to articulate evidence-based decision making. Data on cost effectiveness may be of little use for policy decision making unless it could be contextualised in the territory, and placed within the broader context of health expenditure, CoI and Burden of Illness (BoI). BoD facilitates comparisons across different health condition or disease groupings by using league 
tables or other ranking procedures to set up priorities in allocation of resources and strategic planning. BoD has played a crucial role in priority setting of mental disorders at the country level and worldwide (Murray \& Lopez, 1996). Furthermore, financing and CoI data are also needed in strategic care planning. For example, total expenditure in antidepressants may be a relevant indicator for cost control of the mental health care, but this information should be complemented with cost-of-illness information on the share of use and costs of antidepressants for patients with depression in contact with services in relation to the total health direct costs and - when available - in relation to total costs. The impact of a national programme on prevention of suicide should be analysed both from the financing perspective and from the CoI perspective. CoI may indicate, for example, that mortality costs related to suicide are in a given country at the bottom line of Europe, while morbidity costs related to employment disability benefits are at the highest rate. This information may be critical to allocate a new seed funding in programmes for the prevention of suicide, in programmes for promotion of mental health at the workplace, or in improving the assessment of work-related disabilities.

When a single approach is followed (either FoI, CoI or $\mathrm{BoD}$ ) a partial description is provided and the final information may not only be incomplete but also misleading. Data on financing could be aggregated to CoI data and vice versa, providing imprecise estimate of both costs and expenditures. This is the case of the report on costs of the health programmes in Catalonia, which imputes expenditures of social and health services for dementia and total costs of psychotropic medication to the $\mathrm{MH}$ budget, and produces an overestimate of the $\mathrm{MH}$ share of the total public health expenditure in Catalonia (Spain) (Gisbert et al. 2007).

\section{The steps forward}

In order to advance the monitoring system, a series of alternatives come into view.

First, there is an urgent need for reaching international standard to collect and to compare quantitative economic information. Consensus should initiate by setting the scope of the mental health field, as to which entities include, how to deal with the associated social services and what non-health services to consider. Measurement strategies should indicate the various relevant reporting layers to compare at national and international levels. There are plenty of national and international guidelines for the cost analysis of pharmaceuticals and, hence, international standards are available for any study on cost of alternatives.
However, financing, accounting and CoI studies use a wide range of methods and rules to aggregate data and it is nearly impossible to make any comparison across studies even within a single country, at the required disaggregate information level by disease.

Second, the methods developed to date for service classification and for standard unit cost calculation are complex, time-consuming, heterogeneous and show feasibility problems. The Quatro study on adherence of schizophrenia provides a clear example of these caveats of unit cost calculation in international studies (Patel, 2006). A genuine contribution is required to ensure that mental health becomes feasible on both an epidemiologically and financially monitorable entity.

Third, an explicit review on data collection gathering and administrative records is suggested as feedback to the HIS to improve data sources. For example, there is an urgent need to incorporate Geographical Information Systems (GIS) and spatial analysis to health financing in order to improve the usability of the economic information in evidencebased health care. Mental Health financing and health accountability studies stress the importance of mapping services as a key part of the financing and the accountability processes (WHO, 2003; WHO et al. 2003). On the other hand, cost-effectiveness studies do not usually report characteristics of the area where centres are located. Both socio-demographic characteristics and service availability may influence service use and related costs (Tibaldi et al. 2005). For example, the availability of hospital care in one area may have an influence on hospitalisation and 'relapse' rates of schizophrenia when 'relapse' is related to a pattern of service use (Kane et al. 1998). Hence, both direct and indirect costs may be significantly higher in an area with bed availability far above the median, than in another area without non-acute hospital care.

Fourth, a number of problems may contribute to a systematic bias of measurement in health economic studies and hamper comparisons like-with-like, which should be handled on ad-hoc basis. The terminological variance in service name and coding is a relevant factor in service comparison both in health economics and health financing studies (Zechmeister \& Osterle, 2006). The ICF service classification system (WHO, 2001) is too imprecise to be used for accountability purposes. The International Classification of Health Accounts (ICHA) (OECD, 2009), including their Health providers and Health Functions components have consistency problems and to avoid overlaps and missing relevant content require improvement during the current revision. Even then, clinical practice variation may produce a different pattern of activities in equivalent facilities (Gigantesco 
et al. 2007). Furthermore, the lack of a standard method of unit cost calculation to translate service utilisation into monetary units impedes reliable and valid cost comparison across countries (Patel, 2006; Moreno et al. 2008).

\section{Conclusion}

To improve evidence-based health planning, information on BoD, CoI, FoI, health accounting and cost control should be incorporated to more traditional cost-of-alternatives (cost-effectiveness) data. This information should be also combined and represented using GIS. Mental health care poses similar challenges due to the complexity and the peculiarities of this sector. Three critical aspects for adding these analyses to conventional health economic data are (1) the development of consensus-based guides, indicators and a standard organisation of the available information within a common knowledge base format; (2) the incorporation of modelling to the analysis of data on financing and related issues; and (3) improve knowledge transfer strategies across different stakeholders with regard to health economic evidence.

\section{Acknowledgment}

We are grateful to Jean-Pierre Poullier for his insightful comments. This review has been partly funded by the European Commission under the Executive Agency of Health and Consumers (EAHC) (project eDESDE-LTC Ref. No. 2007116) and the Seventh Framework Programme (project REFINEMENT Ref. No. 261459).

\section{Declaration of Interest}

None

\section{References}

Andlin-Sobocki P, Jönsson B, Wittchen HU, Olesen J (2005). Cost of disorders of the brain in Europe. European Journal of Neurology 12 (Suppl. 1), 1-27.

Ayuso-Mateos JL, Salvador-Carulla L, Chisholm D (2006). Use of quality of life measures in mental health economics and care planning. Actas Espanola de Psiquiatria 34, 1-6.

Byng R, Norman I, Redfern S, Jones R (2008). Exposing the key functions of a complex intervention for shared care in mental health: case study of a process evaluation. BMC Health Services Research 8, 274.

Chisholm D, Stanciole AE, Tan Torres ET, Evans DB (2010). Economic impact of disease and injury: counting what matters. British Medical Journal 340, 924.

Eisenberg L (1973). The future of psychiatry. Lancet ii, 13711375.
Vieth H (2009). Mental health policies in Europe. Euro Observer 11, 3: 1-4.

European Commission (2005). Green Paper: Improving the Mental Health of the Population: Towards a Strategy on Mental Health for the European Union. Health and Consumer Protection Directorate General, European Communities: Brussels.

Evers S, Salvador-Carulla L, Halsteinli V, McDaid D (2007). Implementing mental health economic evaluation evidence: building a bridge between theory and practice. Journal of Mental Health 16, 223-241.

Gigantesco A, Miglio R, Santone G, de Girolamo G, Bracco R, Morosini P, Norcio B, Picardi A, PROGRES Group (2007). Process of care in general hospital psychiatric units: national survey in Italy. Australian and New Zealand Journal of Psychiatry 41, 509-518.

Gisbert R, Brosa M, Bohigas L (2007). Distribución del presupuesto sanitario público de Cataluña del año 2005 entre las 17 categorías CIE-9-MC. Gaceta Sanitaria 21, 12431.

Gold MR, Stevenson D, Fryback DG (2002). HALYs and QALYs and DALYs, Oh My: Similarities and differences in summary measures of population health. Annual Review of Public Health 23, 115-134.

Health Affairs (2007). Prologue. Health care financing worldwide: An overview. Health Affairs (Milwood) 26, 920.

Heurgren M, Arnesson A, André B (2004). A new grouping logic for psychiatric care in NordDRG. Full paper to the 20th International Working Conference on Patient Classification System/Europe (PCS/E) in Budapest, October 2004.

Hu TW (2006). An international review of the national cost estimates of mental illness, 1990-2003. Journal of Mental Health Policy and Economics 9, 3-13.

Jones J, Amaddeo F, Barbui C, Tansella M (2007). Predicting costs of mental health care: a critical literature review. Psychological Medicine 37, 467-477.

Kane JM, Aguglia E, Altamura AC, Ayuso Gutierrez JL, Brunello N, Fleischhacker WW, Gaebel W, Gerlach J, Guelfi JD, Kissling W, Lapierre YD, Lindström E, Mendlewicz J, Racagni G, Carulla LS, Schooler NR (1998). Guidelines for depot antipsychotic treatment in schizophrenia. European Neuropsychopharmacology Consensus Conference in Siena, Italy. European Neuropsychopharmacology 8, 55-66.

Knapp M, McDaid D, Amaddeo F, Constantopoulos A, Oliveira MD, Salvador Carulla L, Zechmeister I, The MHEEN Group (2007). Financing mental health care in Europe. Journal of Mental Health 16, 167-180.

Knapp M, McDaid D, Medeiros H, Becker T, Johnson S, Kilian R, Salvador-Carulla L, Simon J, Tatar M (2008). Economics, Mental Health and Policy: An Overview. MHEEN Policy Briefing. LSE and PSSRU. London School of Economics and Political Science: London (http://eprints.lse. ac.uk/23285/).

McDaid D, Knapp M, Curran C (2006). Meeting the challenge of funding and allocating resources to mental health across Europe: developing the Mental Health Economics European Network. Epidemiologia e Psichiatria Sociale 15, 117-122. 
Mogyorosy Z, Smith P (2005). The Main Methodological Issues in Costing Health Care Services: a Literature Review. Centre for Health Economics Research Paper 7. University of York: York.

Moreno K, Sanchez E, Salvador-Carulla L (2008). Methodological advances in unit cost calculation of psychiatric residential care in Spain. Journal of Mental Health Policy and Economics 11, 79-88.

Murray CJL, Lopez AD (1996). The Global Burden of Disease: a Comprehensive Assessment of Mortality and Disability from Diseases, Injuries, and Risk factors in 1990 and projected to 2020. Harvard University Press: Boston, MA.

Northcott D, Llewellyn S (2004). Decision Making in Health Care: Exploring Cost Variability. CIMA Research Monograph Series 1(2). CIMA Publishing: London.

OECD (2009). The 2010 Joint OECD, EUROSTAT and WHO Health Accounts Data Collection. OECD/HEA/HA(2009)5 (http://www.oecd.org/health/sha/jointquestionnaire). Accessed 14 March 2010.

Orosz E, Morgan D (2004). SHA-Based National Health Accounts in Thirteen OECD Countries: A Comparative Analysis. DELSA/ELSA/WD/HEA (http://www.oecd.org/ dataoecd/10/53/33661480.pdf). Accessed 14 March 2010.

Patel A (2006). Conducting and interpreting multi-national economic evaluations: the measurement of costs. In: Unit Costs of Health and Social Care. Unit Costs of Health and Social Care 2006 (ed. L. Curtis and A. Netten). PSSRU, University of Kent: Canterbury.

Poullier JP, Hernandez P (2001). Health systems, health financing and their measurement. Joint CPC/PAHO/WHO/ FUNSALUD workshop on development of national health accounts in the Caribbean, Bridgetown, 10-14 September 2001.

Rice DP (1966). Estimating the cost of illness. Rockville: Department of Health, Education and Welfare. Health Economic Series No. 6. DHEW Publication No. (PHS) 947-946.

Salvador-Carulla L, Garcia-Alonso C, Gonzalez-Caballero JL, Garrido M (2007). Use of an operational model of community care to assess technical efficiency and benchmarking of small mental health areas in Spain. Journal of Mental Health Policy and Economics 10, 87-100.

Salvador-Carulla L, Haro JM, Ayuso-Mateos JL (2006). A framework for evidence-based mental health care and policy. Acta Psychiatrica Scandinavica 111 (Suppl. 432), 5-11.

Salvador-Carulla L, Saldivia S, Martinez-Leal R, Vicente B, Garcia-Alonso C, Grandon P, Haro JM (2008). Meso-level comparison of mental health service availability and use in Chile and Spain. Psychiatric Services 59, 421-428.
Savedoff W (2007). What should a country spend on health care? Health Affairs (Milwood) 26, 962-970.

Saxena S (2003). Budget and financing of mental health services: baseline information on 89 countries from WHO's Project Atlas. Journal of Mental Health Policy and Economics 6, 135-143.

Thomson S, Foubister T, Mossialos E (2009). Financing Health Care in the European Union: Challenges and Policy Responses. Observatory Studies Series No. 17. World Health Organization: Copenhagen (on behalf of the European Observatory on Health Systems and Policies).

Tibaldi G, Munizza C, Pasian S, Johnson S, Salvador-Carulla L, Zucchi S, Cesano S, Testa C, Scala E, Pinciaroli L (2005). Indicators predicting use of mental health services in Piedmont, Italy. Journal of Mental Health Policy and Economics 8, 95-106.

Trogdon JG, Finkelstein EA, Nwaise IA, Tangka FK, Orenstein D (2007). The economic burden of chronic cardiovascular disease for major insurers. Health Promotion Practice 8, 234-42.

Whitten P, Adams I (2003). Success and failure: a case study of two rural telemedicine projects. Journal of Telemedicine and Telecare 9, 125-129.

World Health Organization (1996). The Ljubljana Charter on Reforming Health Care. WHO Regional Office for Europe: Copenhagen.

World Health Organization (2000). Global Burden of Disease Study. WHO: Geneva.

World Health Organization (2001). International Classification of Functioning, Disability and Health (ICF). WHO: Geneva.

World Health Organization (2003). Mental Health Financing. WHO: Geneve.

World Health Organization (2008a). Policies and Practices for Mental Health in Europe. Meeting the Challenges. WHO Regional Office for Europe: Copenhaghen.

World Health Organization (2008b). Mental Health Gap Action Programme. Scaling up Care for Mental, Neurological, and Substance use Disorders. WHO: Geneva (http://www. who.int/mental_health/mhgap_final_english.pdf).

World Health Organization (2009). WHO Guide to Identifying the Economic Consequences of Disease and Injury. Department of Health Systems Financing. WHO: Geneva.

World Health Organization, World Bank and USAID (2003). Guide to Producing National Health Accounts, with Special Applications for Low-Income and Middle-Income Countries. WHO: Geneva.

Zechmeister I, Osterle A (2006). Distributional Impacts of Mental Health Care Financing Arrangements: A Comparison of the UK, Germany and Austria. Journal of Mental Health Policy and Economics 9, 35-44. 